\title{
A Framework for the Adaptation of Positive Psychological Interventions to North American Indigenous Populations
}

\author{
Melissa R. Schick ${ }^{1}$ (1) $\cdot$ Katelyn T. Kirk-Provencher ${ }^{1} \cdot$ Silvi C. Goldstein $^{1} \cdot$ Tessa Nalven $^{1} \cdot$ Nichea S. Spillane $^{1}$
}

Accepted: 6 July 2021 / Published online: 22 July 2021

(c) Society for Prevention Research 2021

\begin{abstract}
Positive psychology research has led to the development of brief interventions designed to promote positive emotions: positive psychological interventions (PPIs). Randomized controlled trials examining PPIs have found them to be effective in increasing well-being and decreasing depressive symptoms. PPIs have been studied in samples consisting primarily of White Americans; however, PPIs may be useful for members of North American Indigenous groups. PPIs align well with Indigenous views on health, which tend to be strengths-based, holistic, and encompassing the whole body (including the medicine wheel's four dimensions of spirit, mind, heart, and body). This paper provides a framework for the adaptation of PPIs for Indigenous communities and a review of preliminary data on the relationships between positive psychological characteristics and health outcomes including substance use. Implications include the potential widespread impact of culturally adapted PPIs given their alignment with Indigenous thoughts on health and relative ease of administration.
\end{abstract}

Keywords Indigenous · Positive psychology $\cdot$ Positive psychology interventions

This paper calls for efforts to partner with North American Indigenous (NAI) populations using community-based participatory research (CBPR) methods to culturally adapt positive psychological interventions (PPIs). Based on literature, we believe that the principles of positive psychology and Indigenous conceptualizations of health and well-being are well-aligned. We review interventions that, although not making use of existing PPIs, are utilized with NAI populations and are consistent with a positive psychology framework. Finally, we offer suggestions for culturally adapting some existing PPIs.

NAI populations include American Indian (AI) and Alaska Native (AN) people in the USA and First Nation (FN) people in Canada, among others. There are over 500 American Indian tribes and over 600 First Nation communities in Canada. In 2018, 1.3\% of the US population identified as AI/AN (either exclusively, or in combination with some other racial group), making them the second smallest racial/ethnic group in the USA (after Native Hawaiian/Other Pacific Islander; US Census Bureau, 2018). In 2016, 2.9\%

Melissa R. Schick

melissa_schick@uri.edu

1 PATHS Lab, Department of Psychology, University of Rhode Island, 142 Flagg Road, Kingston, RI 02881, USA of the Canadian population identified as First Nation (either exclusively, or in combination with some other Aboriginal group, i.e., Metis or Inuk).

NAI populations have experienced some of the worst health conditions since the arrival of colonists to North America, who introduced smallpox, tuberculosis, measles, influenza, and other deadly ailments that decimated Indigenous communities (Jones, 2006). In fact, a 1585 settler observing the genocide among the Roanok Indigenous people in present-day Virginia remarked "wherever the English visited, the people began to die very fast" (Hariot, 1972). Despite the establishment of the Indian Health Service in the USA in 1955, health disparities persist today. Specifically, death due to diabetes, chronic liver disease, cirrhosis, and accidents occur at least three times more frequently, and deaths due to tuberculosis, pneumonia, influenza, homicide, and heart disease occur at a higher rate among NAI populations compared to non-Indigenous populations (Castor et al., 2006). NAI communities are also disproportionately affected by social determinants of health, including less access to healthy food options and higher unemployment rates (Meyer et al., 2013). Further, NAI communities experience higher rates of mental health concerns, including posttraumatic stress disorder, alcohol dependence, and significantly heightened rates of suicide compared to the general population 
(Meyer et al., 2013; Shalala et al., 1998; Spillane \& Smith, 2010). One proposed explanation for these health disparities is the historical trauma experienced by these communities (Brave Heart et al., 2011).

Historical trauma, or the "legacy of chronic trauma and unresolved grief across generations" (Brave Heart, 1998; Brave Heart \& DeBruyn, 1998), is apparent in NAI peoples, such that they have experienced widespread genocide, legally sanctioned ethnic cleansing, and policies of forced acculturation since first contact with colonizers. These policies led to the criminalization of important cultural practices, which led to the disruption and loss of traditional healing practices, among other effects. It has been proposed that this loss has contributed greatly to the physical and mental health disparities observed among Indigenous populations (Dickerson et al., 2020; Skewes \& Blume, 2019; Whitbeck et al., 2004; Wiechelt et al., 2012). A strengthsbased approach may be effective in addressing these disparities especially given that the effects of historical trauma may stem from the criminalization of culture (Donovan et al., 2015), which held Indigenous culture as being less desirable than mainstream White culture.

\section{Strength-Based Approaches to Mental Health Among NAI Populations}

The medicine wheel (Fig. 1) is an important symbol in many NAI groups (Dapice, 2006). It is represented by a circle including four dimensions of health and well-being that must be balanced and nurtured: spirituality (spirit), mental (mind), emotional (heart), and physical (body;

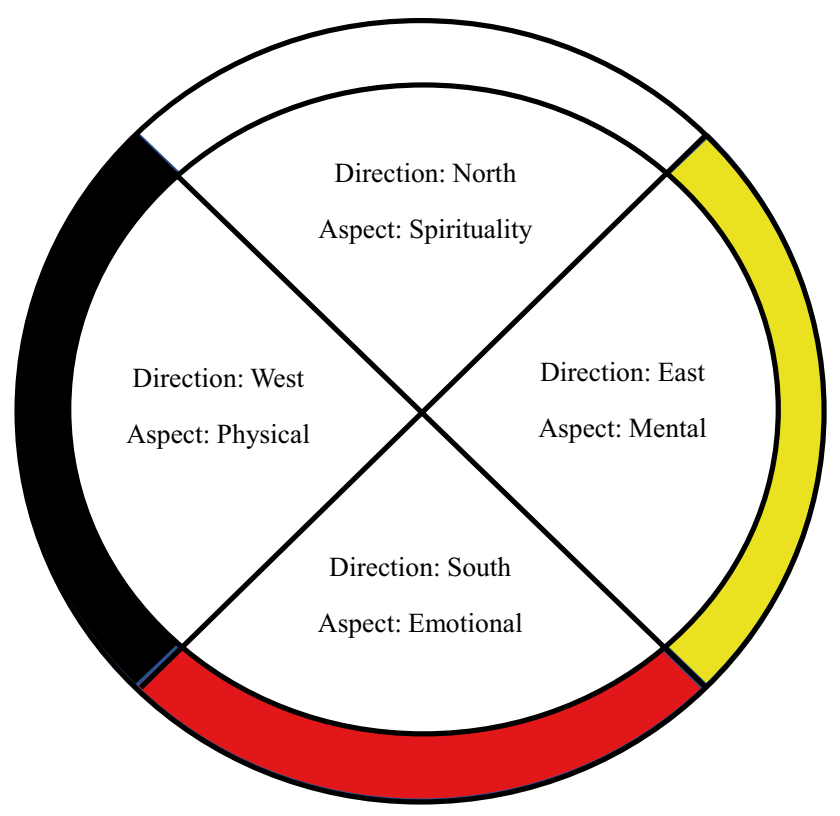

Fig. 1 The Indigenous medicine wheel
Gray, 2012). The wheel represents a holistic view of wellbeing that includes the individual, family, and community and underscores well-being as intrinsically tied to a sense of belonging and purpose within the community (Bear \& Wind, 2011). Further, the medicine wheel is one of the most highly used frameworks for conceptualizing health by NAI groups (Waldram et al., 2006). The emphasis on strengthbased conceptions of well-being, including mental health, is an important aspect of the medicine wheel framework. While many forms of psychotherapy take a deficit approach wherein problems are the focus of treatment, strengths-based approaches stress the positive aspects of an individuals' experience. In NAI communities, health is often conceptualized as being able, holistic, and encompassing the whole body, emphasizing the wholeness of the individual, the interrelatedness of human experiences, and focused on positive communities (Craven et al., 2016). Given the tremendous resilience that NAI populations have shown, strength-based approaches may be used to leverage these strengths.

Positive psychology was originally introduced into the field of psychology as a strengths-based approach to wellbeing during Martin Seligman's 1998 presidential address to the American Psychological Association. He described the field of psychology as having failed in two out of three of the pre-World War II goals to (1) cure mental illness, (2) make the lives of all people more productive and fulfilling, and (3) identify and nurture high levels of talent in individuals. Indeed, psychology seemed to have made progress toward only one of its goals (though it did not achieve the goal): curing mental illness (Seligman \& Csikszentmihalyi, 2000). Yet, it is recognized that well-being is an important component of mental health (World Health Organization, 2005) and that symptom reduction alone is insufficient for flourishing. People can be unhappy and struggle to function in their daily life, yet not meet criteria for any mental illness. Conversely, people can cope effectively with mental illness and live a fulfilling and satisfied life (Keyes, 2007). Toward these outstanding goals, Seligman called for the establishment of a formal field in psychology that aimed to understand positive subjective experiences, individual traits, and positive institutions. Thus, positive psychology is the study of conditions and processes that contribute to human flourishing, resilience, and well-being and the optimal functioning of people, groups, and institutions (Gable \& Haidt, 2005).

Research in the field of positive psychology has led to the creation of positive psychological interventions (PPIs). PPIs are brief, often self-administered, exercises designed to enhance or elicit positive states such as gratitude by increasing one's engagement in positive activities or drawing one's attention to positive experiences (e.g., "Three Good Things"; Seligman et al., 2005) and happiness (e.g., "Random Acts of Kindness"; Lyubomirsky et al., 2005). For example, the "Three Good Things" exercise asks individuals to notice 
positive events by writing down three good things that have happened each day and is intended to teach individuals to notice, remember, and savor the good things in life. Empirical examination of the this exercise found that individuals reported greater happiness immediately post-intervention, and during 1-week, and 1-, 3-, and 6-month follow-ups compared to a control group who completed a neutral writing exercise (Seligman et al., 2005).

PPIs have the benefit of requiring little time and financial resources, are efficacious, and are easy to implement in individual or group settings, or as self-help exercises. Meta-analyses not focusing on PPIs have found evidence supporting the effectiveness of similarly brief interventions to target other areas (e.g., alcohol use, depression, anxiety, obesity; Cape et al., 2010; Sim et al., 2016; Tanner-Smith \& Lipsey, 2015). Further, brief interventions are comparable in effectiveness to longer courses of treatment (Cape et al., 2010), and effects persist over time (Tanner-Smith \& Lipsey, 2015). With respect to PPIs in particular, two metaanalyses reviewed the literature evaluating these brief interventions (e.g., counting blessings, acts of kindness, savoring, best possible self) and indicated overall promising results for PPIs in increasing subjective and psychological well-being and decreasing depressive symptoms (Bolier et al., 2013; Sin \& Lyubomirsky, 2009). Specifically, Sin and Lyubomirsky (2009) reported statistically significant medium effect sizes for well-being (mean $r=0.29$ ) and depression (mean $r=0.31$ ), while Bolier et al. (2013) reported statistically significant small-to-medium effect sizes for subjective well-being (Cohen's $d=0.34$ ) and for depression (Cohen's $d=0.23$ ). Moreover, Bolier et al. (2013) found that at 3-6month follow-ups, the intervention effects on subjective well-being (Cohen's $d=0.22$ ) and psychological wellbeing (Cohen's $d=0.16$ ) remained significant with a small effect size. However, an important consideration is that the majority of samples included in these meta-analyses were comprised primarily of WEIRD ("White, Educated, Industrialized, Rich, and Democratic") samples, which limits the generalizability of findings. Given the ease with which these interventions can be adapted, delivered, and implemented, that these interventions provide long lasting results, and the high degree of concordance between these strengths-based approaches and Indigenous views on health and well-being, it is promising that PPIs can be effectively adapted for NAI populations.

As such, positive psychology may be well-aligned with Indigenous views on health and well-being given that many Indigenous communities place emphasis on the wholeness of the individual, the interrelatedness of human experiences, and the focus on positive communities (Craven et al., 2016) upon which lie the foundations of positive psychology. Although most literature tends to define happiness based on the Western perspective, that is, as the presence of pleasure and absence of pain (Marecek \& Christopher, 2017), it is likely that there is not simply one universal truth regarding what it means for any particular individual to be flourishing (i.e., living within the optimal range of functioning; Fredrickson, 2006). It is possible that being happy and flourishing is dependent upon alignment with one's particular cultural group's values and must be understood within one's cultural context. Thus, the focus on positive communities within PPIs may be especially important in relation to NAI groups, especially those living in reservations/reserve settings given the central role of communal thriving in many NAI groups' views on well-being. Indeed, although tremendous variability exists across individuals and across groups, NAI communities are generally described as being more collectivistic than non-Indigenous groups (i.e., more interdependent, interconnected with one another, and focused on the needs of others within one's community; Beckstein, 2014). Indigenous happiness and flourishing may have more to do with the person as a whole in relation to the health and well-being of the community than among WEIRD populations; thus, PPIs focusing on increasing happiness and flourishing may need to be adapted to meet the more collectivistic nature of some NAI groups. Further, PPIs may be useful in building resilience given previous literature suggesting that experiencing increased positive emotions can contribute to faster recovery from physiological responses to stress (i.e., the "undoing hypothesis"; Fredrickson et al., 2000). Building resilience may be an important component of flourishing (Park, 2012), including for NAI communities given the tremendous adversity they continue to face.

\section{Inclusion of NAI People in PPI Research}

NAI communities have called for strengths-based approaches to health and well-being (LaFromboise \& Low, 1998); thus, PPIs may be uniquely positioned to be adapted for NAI populations. Further, there is a high degree of concordance between pressing health disparities experienced by NAI communities and areas that PPIs have been found to be effective. Specifically, NAI communities experience disproportionate rates of depression, suicidality (Shalala et al., 1998), tobacco use (Spillane et al., 2020b), and cardiovascular disease (Castor et al., 2006). At the same time, PPIs have been found to be feasible and efficacious among non-NAI groups in targeting depression (Rashid, 2015) and suicidality (Huffman et al., 2014), improving likelihood of successful smoking cessation (Kahler et al., 2015), and increasing treatment adherence in cardiovascular disease (Huffman et al., 2014; Nikrahan et al., 2016). Surprisingly, however, it does not appear that PPIs have been formally employed in a systematic way to target these outcomes in NAI communities. Indeed, an important limitation of the existing literature 
examining the efficacy and effectiveness of PPIs is the lack of reporting of the racial composition of samples.

Specifically, an examination of the 57 unique research projects included in the PPI-focused meta-analyses by Sin and Lyubomirsky (2009) and Bolier et al. (2013) reveal that the vast majority of studies fail to include a description of the racial make-up of their samples in their Methods section. Even when racial make-up is mentioned, it is typically presented simply in terms of the percentage (usually the majority) of White participants in the sample. Although it is quite possible that many more of these papers did include Indigenous participants, this lack of reporting makes it unclear. Moreover, only four studies examined in the metaanalyses report inclusion of Indigenous participants: 1.6\% Native American participants (Hurley \& Kwon, 2012), less than $1 \%$ Native American participants (Luthans et al., 2008), 1.4\% Aboriginal participants (Mongrain et al., 2011), and 1 Native American participant (Reed \& Enright, 2006).

The apparent lack of diverse samples may be related to authors taking a universal approach through not including racial descriptors (i.e., a "color blind" approach). This weakness in the literature makes it difficult to conclusively determine whether PPIs will be effective in enhancing wellbeing for NAI populations, as they have been designed for WEIRD samples. There are compelling reasons to believe that PPIs are congruent with NAIs, but these interventions may need to be culturally adapted to ensure congruence with each community by working in partnership with communities to make sure these strategies are in alignment with cultural values while still maintaining their intended purpose.

Literature indicates that adapted treatments are more efficacious when they incorporate values and beliefs from within specific cultures (Benish et al., 2011; Smith et al., 2011). As such, some common positive psychology constructs will likely need to be re-conceptualized before being adapted for NAI groups. For example, satisfaction with social relationships (e.g., family, friends, living partners) is a stronger predictor of satisfaction with life for people from collectivist-oriented cultures (e.g., many Indigenous groups) than for people from individualist cultures (e.g., most Western cultures; Tam et al., 2012). Other research that has extended this work to include a measure of relationship harmony (e.g., the most important people in one's life) suggests that relationship harmony is more important for collectivist group members than those from more individualist groups (Kwan et al., 1997). Additionally, literature has provided a rather narrow, Western definition of social relationships (e.g., defining them by immediate family or important friendships rather than all those individuals with whom one shares a community); thus, such a definition is not readily amenable to more collective groups such as individuals from NAI backgrounds (BigFoot \& Braden, 1998).
However, these are broad generalizations about the values of collectivistic groups, therefore we recommend that before implementing any approach, one works with the community to identify changes that need to be incorporated.

Although there is little argument that social context is important in promoting mental health, an incomplete picture currently exists of how the unique social environment and relationships of NAI communities' influence, or are influenced by, mental health. Previous work narrowly focuses on peers and/or families and tend to use narrow definitions of "family" that often exclude extended and fictive kin (i.e., those who are unrelated to an individual by birth or marriage but have an emotionally significant relationship with an individual such that the relationship takes on characteristics of a familial relationship; Nelson, 2014; Unger et al., 2004) who may figure prominently in the social networks of NAI individuals (MacPhee et al., 1996; Unger et al., 2004). Therefore, culturally sensitive PPIs will benefit from a more inclusive view of the "family," thus allowing for the integration of culture into these strategies.

Although current well-known PPIs (e.g., Three Good Things, savoring) do not appear to have been adapted for NAI populations, interventions with similar goals of increasing one's well-being, positive affect, and other positive states have been developed, adapted, and evaluated with several samples of NAI people. Here, we outline examples of interventions at various stages of development that, although not incorporating PPIs, have recognized the importance of wellbeing and positive affect as worthwhile outcomes.

\section{Canadian First Nations}

The Outdoor Adventure Leadership Experience (OALE; Ritchie et al., 2015) is a youth program designed to promote resilience (defined as "the ability to successfully cope with change or misfortune"), well-being, and connection with "the Good Life" in First Nation youth. The program is designed to be especially culturally relevant and aligned closely with the medicine wheel. Youth participate in a 10-day expedition, during which they engage in activities that are historically consistent with the activities of their ancestors (Ritchie et al., 2015). Results suggested that the program promoted well-being and resilience via establishing external connections to the Creator, their ancestors, other people, plants, animals, and natural elements (Ritchie et al., 2015).

Next, positive behavioral interventions and supports (PBIS) is a is a widely used framework aiming to promote students' quality of life while reducing problematic behaviors through proactive, evidence-based practices that are tailored to fit the needs and values of each particular school. The primary goal is the instruction of prosocial behavioral expectations through shared social values (e.g., "We value 
everyone acting in this way...") rather than punishment of negative behaviors. This focus on reinforcement of positive behaviors aims to change the overall school environment rather than focusing on particular students. Results from studies employing this framework suggest a decrease in the average number of days suspended per student compared to schools not using this framework (Carr et al., 2002). The PBIS framework for building positive school cultures was adapted to be culturally-based to meet the needs of First Nations adolescents (McIntosh et al., 2014). McIntosh et al. (2014) provide a case study of a school in the Northwest Territories that implemented "The Golden Rules." "The Golden Rules," were approved by the Indigenous community, and served as guiding principles for both staff and students within the school; they were clearly and positively stated, founded in alignment with traditional ways of knowing and learning, and were utilized to maintain balance between the spiritual world, people, land, and self. The school implemented PBIS utilizing "The Golden Rules" to increase community engagement within the school (e.g., inviting community members, leaders, elders, and chiefs to biweekly assemblies at the school), and establish a continuum of support for students in need of additional, specialized support. The implementation of the PBIS program was associated with less problematic behaviors that resulted in suspension, and the school environment was described by visitors as purposeful, calm, respectful, and caring during the years following the intervention (McIntosh et al., 2014).

\section{US American Indians}

The "Living in 2 Worlds" (L2W; Kulis et al., 2017) program aims to reduce substance use among American Indian middle school students and is designed to teach adolescents drug resistance and decision-making skills, based on the keepin' it REAL intervention program (Gosin et al., 2003), which was developed for non-AI youth. Although the keepin' it $R E A L$ program has shown promising results in multicultural samples, it has not been found to be effective at preventing alcohol and marijuana use among urban AI youth (Dixon et al., 2007). The L2W program is culturally adapted to provide culturally grounded prevention messaging (e.g., storytelling as a resistance strategy based on oral tradition of passing on history between generations). Results indicated that students in the school randomized to receive the $\mathrm{L} 2 \mathrm{~W}$ program reported significantly lower rates of cigarette use, relatively lower rates of other substance use, and increased connection to their culture (Kulis et al., 2017), which may support healing from the effects of historical trauma.

Next, the "Healing of the Canoe Project" (HOC; Donovan et al., 2015) is a culturally grounded social skills intervention to promote positive youth development. The program aims to prevent substance use and increase cultural connectedness among AI high school students. Youth who participated in this intervention reported higher levels of self-efficacy, optimism, and hope and also possessed more factual knowledge about substance use and reported less engagement in substance use. High schoolers who participated in the intervention also reported a heightened sense of cultural connectedness and cultural identity following the intervention (Donovan et al., 2015).

The "Drum-Assisted Recovery Therapy for Native Americans" (DARTNA; Dickerson et al., 2014) is a tribally adaptable substance use treatment intervention for AI/AN adults that is focused on building connection with culture as a means to reduce substance use and improve well-being. The intervention utilized talking circles and culturally relevant 12 Steps programming, while primarily focusing on "drum behavior therapy." Participants reported spiritual and mood benefits following the program, with significant improvement on measures of meaning, peace, and physical and functional well-being, as well as maintenance of sobriety or reduction in substance use (Dickerson et al., 2014).

Finally, "Motivational Interviewing and Culture for Urban Native American Youth" (MICUNAY; D'Amico et al., 2020) is a culturally-appropriate substance use intervention that integrates motivational interviewing (which has been previously culturally-adapated for use with NAI populations; Venner et al., 2016) with traditional practices based on the Medicine Wheel and aims to empower youth to learn more about their tribal heritage (e.g., incorporating beading, hoop dancing, drumming, storytelling, singing, prayer). Following implementation, results indicated no significant difference between the groups receiving MICUNAY + Cultural Wellness Gatherings (CWGs) versus the group receiving CWGs only with respect to spirituality, happiness, pride in cultural identity, or substance use behaviors (D'Amico et al., 2020). However, it is worth noting that the CWGs, which were offered to both groups and focused on enhancing connection to cultural practices, may have had an effect on these outcomes that was not able to be observed due to the absence of a no-treatment control group.

These aforementioned findings provide preliminary support for the use of interventions promoting strength-based skill building and/or connection with cultural values and practices in promoting well-being, resilience, and other positive outcomes. Therefore, adapting PPIs for use with NAI groups, including with a focus on incorporating connection with culture, is likely to be beneficial. Indeed, NAI individuals have identified enhancing connection with culture and participation in cultural values as an important component of addressing health disparities (Spillane et al., 2020a). Specifically, PPIs may be relatively easy to adapt for these populations by incorporating the values of the Indigenous medicine wheel and the core tenants of the social-ecological framework for prevention. Further, CBPR approaches, which 
emphasize the equitable involvement of community members and organization representatives as key stakeholders throughout the research process (Israel et al., 2005), would likely highlight additional ways that culture may be integrated into these strategies. Moreover, the use of PPIs tends to be cost-effective, requires little time, and they are easy to integrate into other programming to improve outcomes (Bolier et al., 2013; Sin \& Lyubomirsky, 2009), which may lend nicely to being utilized with under-resourced communities.

\section{A Proposed Adaptation of Positive Psychology for NAI Populations}

The social-ecological model has been used by the Centers for Disease Control and Prevention (Centers for Disease Control and Prevention, 2015) as a framework for violence prevention. This model identifies four levels visualized as concentric circles consisting of the individual, relationships, community, and society, which interact with one another to confer risk and/or protection (see Fig. 2). Importantly, this model has been effectively applied in NAI populations to promote positive health outcomes (e.g., lower substance use, depression, suicidality) across all four levels (Henson et al., 2017). Applying the social-ecological framework to PPIs requires us to think about how PPIs can be adapted at each level while taking into account the teachings of the medicine wheel that figures so prominently into many NAI cultural groups. The social-ecological model considers mental health problems such as substance use a complex interplay between individual, relationship, community, and societal factors. Therefore, targeting each factor with PPIs represents a multi-faceted approach to improving mental health and well-being that is consistent with psychological theory and also respects many NAI groups' traditional ways of knowing. It is important to note, however, that there exists tremendous diversity in the cultural characteristics and traditions among NAI groups. As such, use of the following proposed interventions and adaptations must be informed by not only community-specific values, but by the level of acculturation of the individual (Willeto, 2012).

The individual level refers to individual biological and psychological factors, including current and/or future aspirations, personal wellness, positive self-image, and selfefficacy. Programs focusing on the individual level often include individual knowledge, attitudes, and skill building to promote behavior change (Centers for Disease Control and Prevention, 2015). Within more collectivist societies, such as NAI communities, exercises that focus on the individual level are likely to include a component that addresses how improving or focusing on the self can help the larger community. For example, the using signature strengths exercise (Schutte \& Malouff, 2019) includes identifying a personal

Fig. 2 The social-ecological framework of prevention

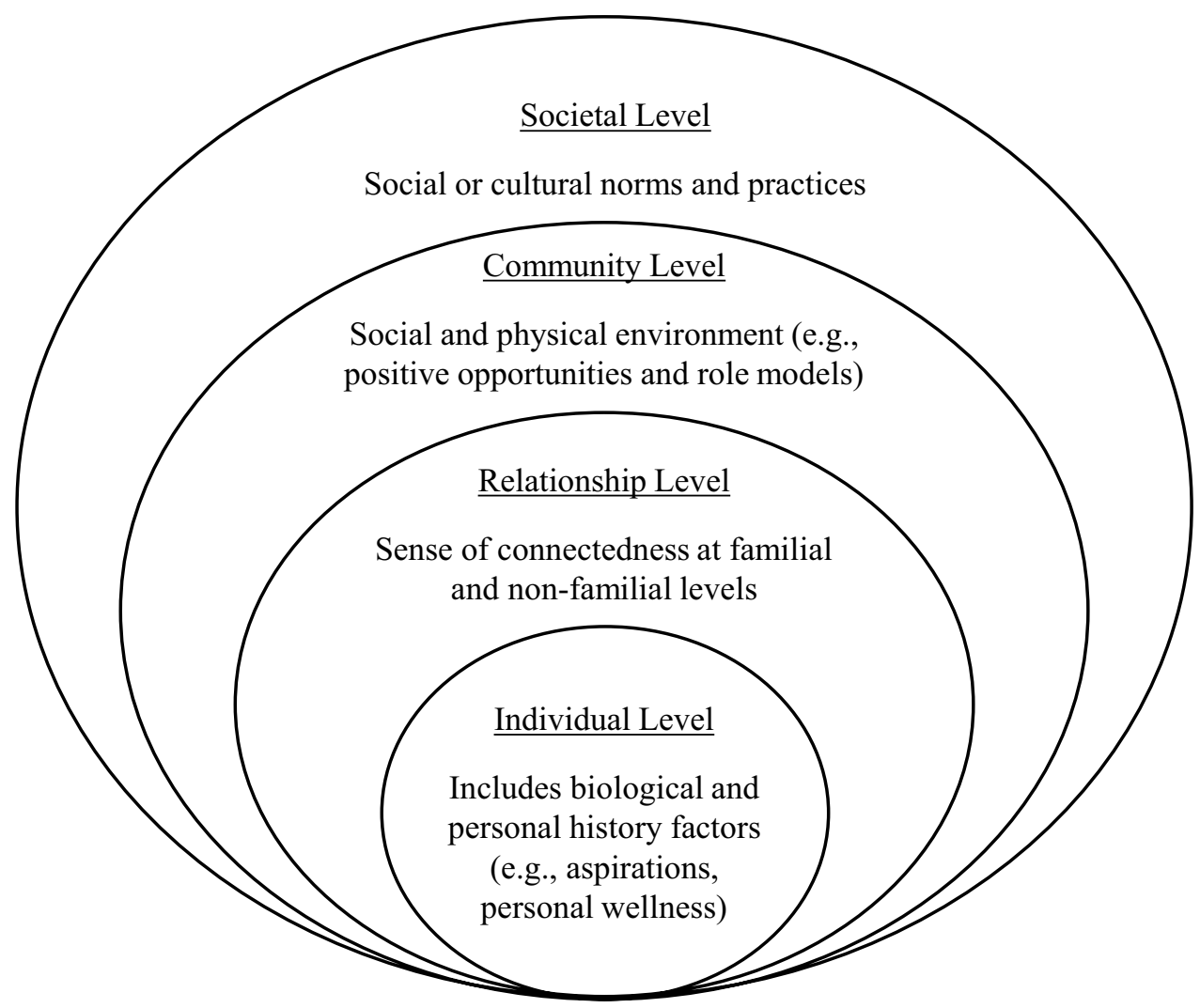


strength and considering how to use it in a new or different way. One consideration for tailoring this activity to be more specific to Indigenous populations could involve using one's strength to benefit the larger community, including extended family or the community where the individual lives. Lovingkindness meditation (Salzberg, 2004) involves meditation focusing on directing loving thoughts towards oneself, then radiating outwards to loved ones, neutral people, and all living beings. This activity appears consistent with the Sweat Lodge ceremony, an important traditional healing activity in many NAI communities (Marsh et al., 2018).

The relationship level is made up of a sense of connectedness at the familial and non-familial levels. Prevention programs focused on this level may include parents or family-focused programs, as well as mentoring and peer programs designed to reduce conflict, foster problem-solving skills, and promote healthy relationships (Centers for Disease Control and Prevention, 2015). Exercises that focus on the relationship level are likely to emphasize caring, support, and recognition of close relationships and guidance received from others. For example, activities focusing on gratitude (e.g., gratitude letters, gratitude visits) involve the individual calling to mind something for which they are extremely grateful, but that they have not properly offered thanks. Then, the individual is instructed to write and deliver a letter to someone or visit someone to express their thanks (Seligman et al., 2005). These types of activities could be adapted to be inclusive of kin and fictive kin (Unger et al., 2004), which may be more in alignment with NAI values. Specifically, as interpersonal relationships are often considered to be particularly important in many NAI communities (Gray, 2012; Spillane et al., 2020a), gratitude activities are likely to align well with existing values and traditions. Further, the positive introduction activity, which asks individuals to tell a story about a time that they were at their best (Seligman et al., 2005), may be reflective of oral traditions around storytelling in NAI communities. This activity could be adapted to focus on the strengths of the collective rather than the individual, such as by asking the individual to share a story about a time that they were proud of their family or community.

The community level is comprised of the social and physical environment, including positive opportunities and positive social norms (e.g., positive adult role models; Centers for Disease Control and Prevention, 2015). Factors that have been identified at the community level that may be protective against mental health difficulties in Indigenous adolescents include positive opportunities (e.g., extra-curricular activities, sports teams) and positive social norms (Henson et al., 2017). Activities focused on this level may include those that encourage individuals to participate in activities that will be of some benefit to their larger community. For example, the Random Acts of Kindness exercise (Lyubomirsky et al., 2005) asks individuals to engage in five acts of kindness throughout one day. Adaptations of this exercise may encourage individuals to consider engaging in acts of kindness that also promote cultural connectedness (e.g., visiting with elders).

The societal level is related to social or cultural norms and policies (Centers for Disease Control and Prevention, 2015). Literature has demonstrated strong evidence for the role of identification with one's Indigenous heritage, involvement in cultural activities, and involvement in traditional spirituality in protecting against mental health concerns (Whitbeck et al., 2001). Incorporation of strengths-based PPIs adapted to emphasize this important cultural connectedness, across other social-ecological levels may prove to promote resilience, happiness, and flourishing. This connectedness may be a helpful component towards the healing process from historical, intergenerational traumas, as it will incorporate important aspects of traditional culture that has been identified by literature and many communities as healing.

\section{Implications for Future Research}

We have provided many examples of possible adaptations and ways to incorporate PPIs to promote NAI mental health that is consistent with the teaching of the medicine wheel by taking a social-ecological approach. Such approaches are urgently needed in part because of the current deficit-focus in treatment models that are largely inconsistent with NAI values. PPIs have been suggested as a useful, less stigmatizing approach to enhance treatment engagement and as an adjunct to problem-focused intervention approaches (Bolier et al., 2013), perhaps because they may bind with existing interventions to promote their efficacy. For instance, when adapted in partnership with NAI populations, PPIs may help individuals reflect on traditional, cultural activities with more gratitude, and therefore, value those activities more. This effect would be of great importance, as valuing cultural activities has been found to be protective against engagement in health risk behaviors among NAI adolescents (Goldstein et al., 2021).

At the same time, it is important to remember that there is tremendous diversity that exists within NAI groups. Thus, specific cultural considerations must be made prior to implementing PPIs with any particular group. We encourage researchers, clinicians, and community leaders to utilize CBPR methods and involve the community in all aspects of adaptation. Alternatively, the Culturally Specific Prevention Framework was developed based on work within NAI communities and calls for substantial community engagement prior to, during, and after a cultural adaptation is undertaken (Whitbeck, 2006). Efforts should aim to include both surface (e.g., intervention materials) and deep structure (e.g., 
incorporating specific cultural values) adaptations that will increase the cultural relevance and efficacy of these adaptations (Knight et al., 2009). Additionally, future research may benefit from examining the effect of PPIs on building resilience and how PPIs can be adapted for this goal given previous research indicating unique cultural pathways to resilience (Anderson, 2019; Ungar et al., 2007). Ultimately, it is important to remember that NAI people must be regarded as the experts on adaptations that are appropriate for members of their communities and thus have full agency and voice in treatment adaptations.

We encourage researchers to investigate these adapted interventions and to empirically assess whether they promote positive mental health outcomes in NAI people. PPIs are easy exercises to engage in and require little time and resources, thus they have the potential for broad outreach and appeal. Thus, PPIs are a feasible approach to promoting NAI mental wellness.

Author Contributions Authors Schick and Spillane designed the study, conducted literature searches, and wrote the initial draft of the manuscript. Authors Kirk-Provencher, Nalven, and Goldstein conducted literature searches and extensively edited and reviewed the manuscript. All authors contributed to and have approved the final version of the manuscript for submission

Funding Work on this paper was supported by National Institute of Alcohol Abuse and Alcoholism grant R34AA027619.

\section{Declarations}

Ethics Approval Ethics approval was not required for this review/theoretical manuscript.

Informed Consent Not applicable—not human subjects research.

Conflict of Interest The authors declare no competing interests.

\section{References}

Anderson, L. A. (2019). Rethinking resilience theory in african american families: Fostering positive adaptations and transformative social justice. Journal of Family Theory \& Review, 11, 385-397. https://doi.org/10.1111/jftr.12343

Bear, S., \& Wind, W. (2011). The medicine wheel: Earth astrology. Simon and Schuster.

Beckstein, A. (2014). Native American subjective happiness: An overview. Indigenous Policy Journal, 25.

Benish, S. G., Quintana, S., \& Wampold, B. E. (2011). Culturally adapted psychotherapy and the legitimacy of myth: A directcomparison meta-analysis. Journal of Counseling Psychology, 58, 279-289. https://doi.org/10.1037/a0023626

BigFoot, D., \& Braden, J. (1998). On the back of a turtle. University of Oklahoma Health Sciences Center.

Bolier, L., Haverman, M., Westerhof, G. J., Riper, H., Smit, F., \& Bohlmeijer, E. (2013). Positive psychology interventions: A meta-analysis of randomized controlled studies. BMC Public Health, 13, 119-138. https://doi.org/10.1186/1471-2458-13-119

Brave Heart, M. Y. H. (1998). The return to the sacred path: Healing the historical trauma and historical unresolved grief response among the lakota through a psychoeducational group intervention. Smith College Studies in Social Work, 68, 287-305. https://doi. org/10.1080/00377319809517532

Brave Heart, M. Y. H., Chase, J., Elkins, J., \& Altschul, D. B. (2011) Historical trauma among indigenous peoples of the americas: Concepts, research, and clinical considerations. Journal of Psychoactive Drugs, 43, 282-290. https://doi.org/10.1080/02791072. 2011.628913

Brave Heart, M. Y. H., \& DeBruyn, L. M. (1998). The American Indian holocaust: Healing historical unresolved grief. American Indian and Alaska Native Mental Health Research, 8, 56-78. https://doi. org/10.5820/aian.0802.1998.60

Cape, J., Whittington, C., Buszewicz, M., Wallace, P., \& Underwood, L. (2010). Brief psychological therapies for anxiety and depression in primary care: Meta-analysis and meta-regression. $B M C$ Medicine, 8, 1-13. https://doi.org/10.1186/1741-7015-8-38

Carr, E. G., Dunlap, G., Horner, R. H., Koegel, R. L., Turnbull, A. P., Sailor, W., Anderson, J. L., Albin, R. W., Koegel, L. K., \& Fox, L. (2002). Positive behavior support: Evolution of an applied science. Journal of Positive Behavior Interventions, 4, 4-16. https:// doi.org/10.1177/109830070200400102

Castor, M. L., Smyser, M. S., Taualii, M. M., Park, A. N., Lawson, S. A., \& Forquera, R. A. (2006). A nationwide population-based study identifying health disparities between American Indians/ Alaska Natives and the general populations living in select urban counties. American Journal of Public Health, 96, 1478-1484. https://doi.org/10.2105/AJPH.2004.053942

Centers for Disease Control and Prevention. (2015). The social-ecological model: A framework for prevention. https://www.cdc.gov/ violenceprevention/about/social-ecologicalmodel.html

Craven, R. G., Ryan, R. M., Mooney, J., Vallerand, R. J., Dillon, A., Blacklock, F., \& Magson, N. (2016). Toward a positive psychology of indigenous thriving and reciprocal research partnership model. Contemporary Educational Psychology, 47, 32-43. https:// doi.org/10.1016/j.cedpsych.2016.04.003

D’Amico, E. J., Dickerson, D. L., Brown, R. A., Johnson, C. L., Klein, D. J., \& Agniel, D. (2020). Motivational interviewing and culture for urban Native American youth (micunay): A randomized controlled trial. Journal of Substance Abuse Treatment, 111, 86-99. https://doi.org/10.1016/j.jsat.2019.12.011

Dapice, A. N. (2006). The medicine wheel. Journal of Transcultural Nursing, 17, 251-260. https://doi.org/10.1177/1043659606288383

Dickerson, D., Baldwin, J. A., Belcourt, A., Belone, L., Gittelsohn, J., Kaholokula, J. K. A., Lowe, J., Patten, C. A., \& Wallerstein, N. (2020). Encompassing cultural contexts within scientific research methodologies in the development of health promotion interventions. Prevention Science, 21, 33-42. https://doi.org/10.1007/s11121-018-0926-1.

Dickerson, D. L., Venner, K. L., Duran, B., Annon, J. J., Hale, B., \& Funmaker, G. (2014). Drum-assisted recovery therapy for Native Americans (dartna): Results from a pretest and focus groups. American Indian and Alaska Native Mental Health Research, 21, 35. https://doi.org/10.5820/aian.2101.2014.35

Dixon, A. L., Yabiku, S. T., Okamoto, S. K., Tann, S. S., Marsiglia, F. F., Kulis, S., \& Burke, A. M. (2007). The efficacy of a multicultural prevention intervention among urban American Indian youth in the southwest us. The Journal of Primary Prevention, 28, 547-568. https://doi.org/10.1007/s10935-007-0114-8

Donovan, D. M., Thomas, L. R., Sigo, R. L. W., Price, L., Lonczak, H., Lawrence, N., Ahvakana, K., Austin, L., Lawrence, A., \& Price, J. (2015). Healing of the canoe: Preliminary results of a culturally grounded intervention to prevent substance abuse and promote tribal identity for native youth in two pacific northwest tribe. 
American Indian and Alaska Native Mental Health Research, 22, 42-76.https://doi.org/10.5820/aian.2201.2015.42

Fredrickson, B. L. (2006). Unpacking positive emotions: Investigating the seeds of human flourishing. The journal of positive psychology, 1, 57-59. https://doi.org/10.1080/17439760500510981

Fredrickson, B. L., Mancuso, R. A., Branigan, C., \& Tugade, M. M. (2000). The undoing effect of positive emotions. Motivation and emotion, 24, 237-258. https://doi.org/10.1023/A: 1010796329158

Gable, S. L., \& Haidt, J. (2005). What (and why) is positive psychology? Review of General Psychology, 9, 103-110. https://doi. org/10.1037/1089-2680.9.2.103

Goldstein, S. C., Schick, M. R., Nalven, T., \& Spillane, N. S. (2021). Valuing cultural activities moderating the association between alcohol expectancies and alcohol use among First Nation adolescents. Journal of Studies on Alcohol and Drugs, 82, 112-120. https://doi.org/10.15288/jsad2021.82.112

Gosin, M., Marsiglia, F. F., \& Hecht, M. L. (2003). Keepin'it real: A drug resistance curriculum tailored to the strengths and needs of pre-adolescents of the southwest. Journal of Drug Education, 33, 119-142. https://doi.org/10.2190/DXB9-1V2P-C27J-V69V

Gray, J. S. (2012). Cultural adaptations for American Indian clients. In G. Bernal \& M. M. D. Rodriguez (Eds.), Cultural adaptations: Tools for evidence-based practice with diverse populations (pp. 361-368). American Psychological Association. https://doi.org/10.1037/a0016401

Hariot, T. (1972). 1588. A briefe and true report of the new found land of virginia. The Roanoke voyages, 1584, 1590.

Henson, M., Sabo, S., Trujillo, A., \& Teufel-Shone, N. (2017). Identifying protective factors to promote health in American Indian and Alaska Native adolescents: A literature review. The journal of primary prevention, 38, 5-26. https://doi.org/10.1007/ s10935-016-0455-2

Huffman, J. C., DuBois, C. M., Healy, B. C., Boehm, J. K., Kashdan, T. B., Celano, C. M., Denninger, J. W., \& Lyubomirsky, S. (2014). Feasibility and utility of positive psychology exercises for suicidal inpatients. General Hospital Psychiatry, 36, 88-94. https://doi. org/10.1016/j.genhosppsych.2013.10.006

Hurley, D. B., \& Kwon, P. (2012). Results of a study to increase savoring the moment: Differential impact on positive and negative outcomes. Journal of Happiness Studies, 13, 579-588. https://doi.org/10.1007/s10902-011-9280-8

Israel, B. A., Eng, E., Schulz, A. J., \& Parker, E. A. (2005). Methods in community-based participatory research for health. Jossey-Bass.

Jones, D. S. (2006). The persistence of American Indian health disparities. American Journal of Public Health, 96, 2122-2134. https:// doi.org/10.2105/AJPH.2004.054262

Kahler, C. W., Spillane, N. S., Day, A. M., Cioe, P. A., Parks, A., Leventhal, A. M., \& Brown, R. A. (2015). Positive psychotherapy for smoking cessation: A pilot randomized controlled trial. Nicotine \& Tobacco Research, 17, 1385-1392. https://doi.org/10.1093/ntr/ntv011

Keyes, C. L. (2007). Promoting and protecting mental health as flourishing: A complementary strategy for improving national mental health. American Psychologist, 62, 95-108. https://doi.org/10. 1037/0003-066X.62.2.95

Knight, G. P., Roosa, M. W., \& Umaña-Taylor, A. J. (2009). Putting research into action: Prevention intervention research. In Studying ethnic minority and economically disadvantaged populations: Methodological challenges and best practices (pp. 167-190). American Psychological Association. https://doi.org/10.1037/ 11887-000

Kulis, S. S., Ayers, S. L., \& Harthun, M. L. (2017). Substance use prevention for urban American Indian youth: A efficacy trial of the culturally adapted living in 2 worlds program. The Journal of Primary Prevention, 38, 137-158. https://doi.org/10.1007/ s10935-016-0461-4

Kwan, V. S., Bond, M. H., \& Singelis, T. M. (1997). Pancultural explanations for life satisfaction: Adding relationship harmony to self-esteem. Journal of Personality and Social Psychology, 73, 1038-1051. https://doi.org/10.1037/0022-3514.73.5.1038

LaFromboise, T., \& Low, K. G. (1998). American Indian children and adolescents. In J. T. Gibbs \& L. N. Huang (Eds.), Children of color: Psychological interventions with culturally diverse youth (pp. 112-142). Jossey-Bass.

Luthans, F., Avey, J. B., \& Patera, J. L. (2008). Experimental analysis of a web-based training intervention to develop positive psychological capital. Academy of Management Learning \& Education, 7, 209-221. https://doi.org/10.5465/amle.2008.32712618

Lyubomirsky, S., Sheldon, K. M., \& Schkade, D. (2005). Pursuing happiness: The architecture of sustainable change. Review of General Psychology, 9, 111-131. https://doi.org/10.1037/1089-2680.9.2.111

MacPhee, D., Fritz, J., \& Miller-Heyl, J. (1996). Ethnic variations in personal social networks and parenting. Child Development, 67, 3278-3295. https://doi.org/10.1111/j.1467-8624.1996. tb01914.x

Marecek, J., \& Christopher, J. C. (2017). Is positive psychology an indigenous psychology? In The routledge international handbook of critical positive psychology (pp. 84-98). Routledge.

Marsh, T. N., Marsh, D. C., Ozawagosh, J., \& Ozawagosh, F. (2018). The sweat lodge ceremony: A healing intervention for intergenerational trauma and substance use. International Indigenous Policy Journal, 9. https://doi.org/10.18584/iipj.2018.9.2.2

McIntosh, K., Moniz, C., Craft, C. B., Golby, R., \& SteinwandDeschambeault, T. (2014). Implementing school-wide positive behavioural interventions and supports to better meet the needs of indigenous students. Canadian Journal of School Psychology, 29, 236-257. https://doi.org/10.1177/0829573514542217

Meyer, P. A., Yoon, P. W., \& Kaufmann, R. B. (2013). Introduction: CDC health disparities and inequalities report-united states, 2013. MMWR Supplements, 62(3), 3-5.

Mongrain, M., Chin, J. M., \& Shapira, L. B. (2011). Practicing compassion increases happiness and self-esteem. Journal of Happiness Studies, 12, 963-981. https://doi.org/10.1007/ s10902-010-9239-1

Nelson, M. K. (2014). Whither fictive kin? Or, what's in a name? Journal of Family Issues, 35, 201-222. https://doi.org/10.1177/ $0192513 X 12470621$

Nikrahan, G. R., Suarez, L., Asgari, K., Beach, S. R., Celano, C. M., Kalantari, M., Abedi, M. R., Etesampour, A., Abbas, R., \& Huffman, J. C. (2016). Positive psychology interventions for patients with heart disease: A preliminary randomized trial. Psychosomatics, 57, 348-358. https://doi.org/10.1016/j.psym. 2016.03.003

Park, N. (2012). Adversity, resilience, and thriving: A positive psychology perspective on research and practices. In R. A. McMackin, E. Newman, J. M. Fogler, \& T. M. Keane (Eds.), Trauma therapy in context: The science and craft of evidencebased practice (pp. 121-140). American Psychological Association. https://doi.org/10.1037/13746-000

Rashid, T. (2015). Positive psychotherapy: A strength-based approach. The Journal of Positive Psychology, 10, 25-40. https://doi.org/10.1080/17439760.2014.920411

Reed, G. L., \& Enright, R. D. (2006). The effects of forgiveness therapy on depression, anxiety, and posttraumatic stress for women after spousal emotional abuse. Journal of Consulting and Clinical Psychology, 74, 920-929. https://doi.org/10.1037/ 0022-006X.74.5.920

Ritchie, S. D., Wabano, M. J., Corbiere, R. G., Restoule, B. M., Russell, K. C., \& Young, N. L. (2015). Connecting to the good life through outdoor adventure leadership experiences designed for indigenous 
youth. Journal of Adventure Education and Outdoor Learning, 15, 350-370. https://doi.org/10.1080/14729679.2015.1036455

Salzberg, S. (2004). Loving-kindness: The revolutionary art of happiness. Shambhala Publications.

Schutte, N. S., \& Malouff, J. M. (2019). The impact of signature character strengths interventions: A meta-analysis. Journal of Happiness Studies, 20, 1179-1196. https://doi.org/10.1007/ s10902-018-9990-2

Seligman, M. E., \& Csikszentmihalyi, M. (2000). Positive psychology: An introduction. American Psychologist, 55, 5-14. https://doi.org/ 10.1037/0003-066X.55.1.5

Seligman, M. E., Steen, T. A., Park, N., \& Peterson, C. (2005). Positive psychology progress: Empirical validation of interventions. American Psychologist, 60, 410-421. https://doi.org/10.1037/ 0003-066X.60.5.410

Shalala, D., Trujillo, M., Hartz, G., \& D’Angelo, A. (1998). Indian health service: Regional differences in indian health. US Government Printing Office.

Sim, L. A., Lebow, J., Wang, Z., Koball, A., \& Murad, M. H. (2016). Brief primary care obesity interventions: A meta-analysis. Pediatrics, 138, e20160149. https://doi.org/10.1542/peds.2016-0149

Sin, N. L., \& Lyubomirsky, S. (2009). Enhancing well-being and alleviating depressive symptoms with positive psychology interventions: A practice-friendly meta-analysis. Journal of Clinical Psychology, 65, 467-487. https://doi.org/10.1002/jclp.20593

Skewes, M. C., \& Blume, A. W. (2019). Understanding the link between racial trauma and substance use among American Indians. American Psychologist, 74, 88-100. https://doi.org/10.1037/amp0000331

Smith, T. B., Rodríguez, M. D., \& Bernal, G. (2011). Culture. Journal of Clinical Psychology, 67, 166-175. https://doi.org/10.1002/jclp.20757

Spillane, N. S., Kirk-Provencher, K. T., Schick, M. R., Nalven, T., Goldstein, S. C., \& Kahler, C. W. (2020a). Identifying competing life reinforcers for substance use in First Nation adolescents. Substance Use \& Misuse, 55, 886-895. https://doi.org/10.1080/ 10826084.2019.1710206

Spillane, N. S., Treloar Padovano, H., \& Schick, M. R. (2020b). Regional and gender differences in tobacco use among American Indian youth. Journal of Ethnicity in Substance Abuse, 19, 553-566. https://doi.org/10.1080/15332640.2018.1548321

Spillane, N. S., \& Smith, G. T. (2010). Individual differences in problem drinking among tribal members from one First Nation community. Alcoholism: Clinical and Experimental Research, 34, 1985-1992. https://doi.org/10.1111/j.1530-0277.2010.01288.x

Tam, K. P., Lau, H. P. B., \& Jiang, D. (2012). Culture and subjective well-being: A dynamic constructivist view. Journal of Cross-Cultural Psychology, 43, 23-31. https://doi.org/10. 1177/0022022110388568

Tanner-Smith, E. E., \& Lipsey, M. W. (2015). Brief alcohol interventions for adolescents and young adults: A systematic review and meta-analysis. Journal of Substance Abuse Treatment, 51, 1-18. https://doi.org/10.1016/j.jsat.2014.09.001

Ungar, M., Brown, M., Liebenberg, L., Othman, R., Kwong, W., Armstrong, M., \& Gilgun, J. (2007). Unique pathways to resilience across cultures. Adolescence, 42, 166.

Unger, J. B., Baezconde-Garbanati, L., \& Soto, C. (2004). Family-and peer-related risk and protective factors for tobacco use among American Indian adolescents in California. Journal of Ethnicity in Substance Abuse, 3, 1-15. https://doi.org/10.1300/J233v03n04_01

US Census Bureau. (2018). 2018 quick facts. https://www.census.gov/ quickfacts/fact/table/US/PST045219

Venner, K. L., Greenfield, B. L., Hagler, K. J., Simmons, J., Lupee, D., Homer, E., Yamutewa, Y., \& Smith, J. E. (2016). Pilot outcome results of culturally adapted evidence-based substance use disorder treatment with a southwest tribe. Addictive Behaviors Reports, 3, 21-27. https://doi.org/10.1016/j.abrep.2015.11.002

Waldram, J. B., Herring, A., \& Young, T. K. (2006). Aboriginal health in canada: Historical, cultural, and epidemiological perspectives (2nd ed.). University of Toronto Press.

Whitbeck, L. B. (2006). Some guiding assumptions and a theoretical model for developing culturally specific preventions with Native American people. Journal of Community Psychology, 34, 183192. https://doi.org/10.1002/jcop.20094

Whitbeck, L. B., Chen, X., Hoyt, D. R., \& Adams, G. W. (2004). Discrimination, historical loss and enculturation: Culturally specific risk and resiliency factors for alcohol abuse among American Indians. Journal of Studies on Alcohol, 65, 409-418. https://doi. org/10.15288/jsa.2004.65.409

Whitbeck, L. B., Hoyt, D. R., Stubben, J. D., \& LaFromboise, T. (2001). Traditional culture and academic success among american indian children in the upper midwest. Journal of American Indian Education, 40, 48-60.

Wiechelt, S. A., Gryczynski, J., Johnson, J. L., \& Caldwell, D. (2012). Historical trauma among urban american indians: Impact on substance abuse and family cohesion. Journal of Loss and Trauma, 17, 319-336. https://doi.org/10.1080/15325024.2011.616837

Willeto, A. A. (2012). Happiness in navajos (diné ba'hózhó). In Happiness across cultures (pp. 377-386). Springer.

World Health Organization. (2005). Promoting mental health: Concepts, emerging evidence, practice: A report of the world health organization, department of mental health and substance abuse in collaboration with the victorian health promotion foundation and the university of melbourne. World Health Organization.

Publisher's Note Springer Nature remains neutral with regard to jurisdictional claims in published maps and institutional affiliations. 